\title{
Polarimetric Analysis of Scatterometer Data for Ocean Surface Wind Measurement
}

\author{
J.S. Lee ${ }^{1}$, S. H. Yueh ${ }^{2}$ and D.L. Schuler ${ }^{1}$ \\ ${ }^{1}$ Naval Research Laboratory, Washington DC 20375-5351 \\ ${ }^{2}$ Jet Propulsion Laboratory, MS 300-235, California Institute of Technology, Pasadena, CA 91109
}

\begin{abstract}
An experiment using a polarimetric scatterometer (POLSCAT) has been conducted by JPL for ocean surface wind measurement. It shows that $\sigma_{0}$ values for $\mathrm{HH}, \mathrm{VV}, \mathrm{HV}$, and $\mathrm{VH}$ have the property of even symmetry with respect to the upwind direction, and correlation coefficients between co- and crosspolarizations have the odd symmetry property. In this paper, the symmetry properties will be further examined using polarimetric analysis to investigate the depolarization effect, the scattering mechanism, and the polarization orientation angle. Theoretical results based on a two scale model are used to verify the derived experiment results. The newly derived symmetry property has the potential to solve the $180^{\circ}$ ambiguity in wind direction, and to enhance the accuracy of wind vector measurements.
\end{abstract}

Keywords- Scatterometer;Radar Polarimetry; Ocean Wind

\section{INTRODUCTION}

The first experiment using a polarimetric scatterometer (POLSCAT) for ocean surface wind measurements was successfully conducted by Yueh and Wilson [1] in August 2000. The airborne POLSCAT, developed by JPL, measures ocean surface backscatter at $\mathrm{Ku}$-band $(13.95 \mathrm{GHz})$ with quadpolarizations $(\mathrm{HH}, \mathrm{HV}, \mathrm{VH}$ and $\mathrm{VV})$. A set of flights over buoys off the California coast has been performed to investigate the dependence and sensitivity of polarimetric radar cross-sections with respect to the wind direction. The measured HV and VH echoes are nearly the same, demonstrating the reciprocal relationship for radar backscatter. The normalized cross-section, or $\sigma_{0}$ values for all four polarizations show even-symmetry properties with respect to the upwind direction, but the correlation between co- and cross-polarization radar returns has an odd-symmetry, which could help to solve the 180 degree wind direction ambiguity that typically exists in scatterometer wind retrievals.

In this paper, we will apply polarimetric analysis techniques to further investigate the symmetry properties of the depolarization effect, the polarization orientation angle, the polarimetric parameters from Cloude's target decomposition [4], and circular polarization returns. A theoretical two- scale wave model is used to verify the derived experimental results. We believe that this study will provide a theoretical explanation of the useful odd-symmetry property in co- and cross-polarization correlation, and that newly derived symmetry properties can be used to enhance the accuracy of wind vector measurements.

In two scale models, it is known that hydrodynamic modulation makes the short waves more concentrated on the leeward faces of large-scale waves, and that this induces higher returns in upwind direction than in the downwind direction. Slopes associated with small and large-scale waves have a symmetrical distribution that induces reflection symmetry [2] in polarimetric covariance matrices for the upwind and downwind direction. However, when the radar looks into the crosswind direction, the difference between returns from the leeward slope and the windward slope causes shifts in the polarization orientation angle [3], and produces reflection asymmetry. We analyzed the JPL POLSCAT data and found that, as expected, the shifts in orientation angles in the upwind and downwind directions are near zero, but peaks occurred near $\pm 60^{\circ}$ from the upwind direction. The orientation angle can be used as a measure of azimuth slopes. Peak-to-peak azimuth slopes of 4 degrees are observed. Since there is a coarse spatial resolution associated with the scatterometer, several long waves are included in a resolution cell. The symmetry properties are investigated using a simple two-scale wave model. The theoretical results seem to confirm the experimental results.

The depolarization effect is investigated using the entropy value of the eigenvector based scattering decomposition proposed by Cloude and Pottier [4]. The entropy value is peaked at crosswind directions revealing surface scattering is weaker and the contribution of double bounce scattering is more significant. Other polarimetric parameters, such as, alpha angle, anisotropy, and circular polarizations have also been investigated.

\section{POLARIMETRIC ANALYSIS}

All POLSCAT data sets collected in the August 2000 experiment have the same polarimetric characteristics. We use the M2 Aug 16 data as an example. The average wind speed during the 1-hour flight of ten passes, in a star pattern over a buoy, was about $10.8 \mathrm{~m} / \mathrm{s}$. The $\sigma_{0}$ of $\mathrm{HH}$ and $\mathrm{VV}$ polarization is shown in the top plot of Fig. 1, and $\mathrm{HV}$ and $\mathrm{VH}$ is shown is shown in the middle plot. The upwind direction corresponds to an azimuth angle of $0^{\circ}$. The $\sigma_{0}$ values show even symmetry as indicated by Yueh and Wilson [1]. The correlation coefficient between $\mathrm{VV}$ and $\mathrm{HV}$ is shown in the lower plot revealing odd-symmetry in both real and imaginary parts. The coefficients between HH nad HV is similar.

Yueh and Wilson [1] pointed out that the peaks of $\mathrm{VV}$ and $\mathrm{HV}$ correlation are located around $\pm 60^{\circ}$ rather than $\pm 120^{\circ}$ as predicted in their theoretical two-scale model. We also 
noticed secondary peaks located near $\pm 120^{\circ}$ as marked by arrows in the lower plot of Fig. 1. The discrepancy of the major peaks' locations and the existance of secondary peaks will be explained using a theoretical two-scale model.
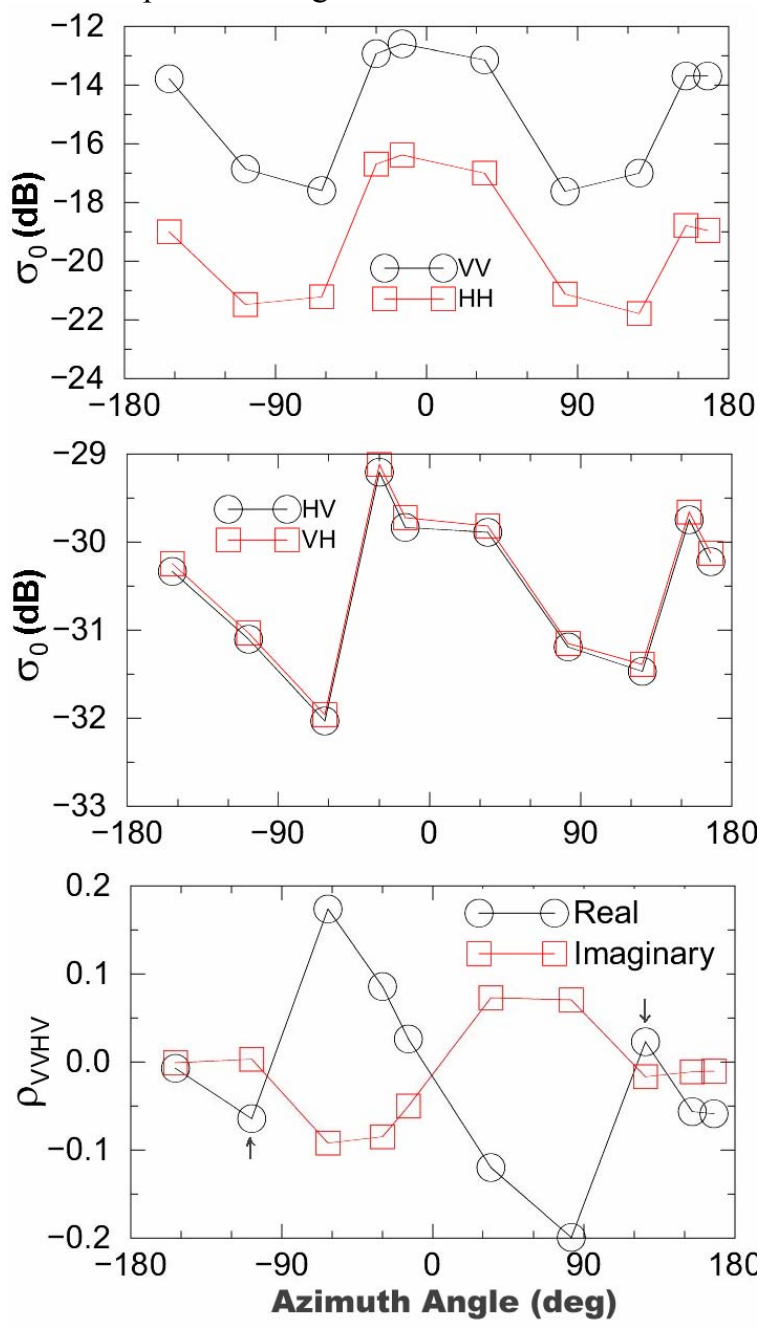

Fig. 1 POLSCAT data versus radar look direction. The upwind direction corresponds to the azimuth angle of the value $0^{\circ}$.

\section{A. Polarization orientation angle}

Azimuthal slopes of long waves can induce a polarization orientation angle shift, which can be measured by the $\boldsymbol{R} \boldsymbol{R}$ (right-right) and $\boldsymbol{L} \boldsymbol{L}$ (left-left) circular polarization phase difference [3]. The $\boldsymbol{R} \boldsymbol{R}, \boldsymbol{L} \boldsymbol{L}$ and $\boldsymbol{R} \boldsymbol{L}$ circular polarizations can be obtained by

$$
\begin{aligned}
& S_{R R}=\left(S_{H H}-S_{V V}+i 2 S_{H V}\right) / 2 \\
& S_{L L}=\left(S_{V V}-S_{H H}+i 2 S_{H V}\right) / 2 \\
& S_{R L}=i\left(S_{H H}+S_{V V}\right) / 2
\end{aligned}
$$

where $\boldsymbol{S}_{\boldsymbol{H} \boldsymbol{V}}$ is the complex return of $\boldsymbol{H} \boldsymbol{V}$. The orientation angle, $\theta$, is obtained by

$$
\theta=\left\{\begin{array}{crr}
\eta, & \text { if } & \eta \leq \pi / 4 \\
\eta-\pi / 2, & \text { if } & \eta>\pi / 4
\end{array}\right.
$$

$$
\eta=\frac{1}{4}\left[\tan ^{-1}\left(\frac{-4 \operatorname{Re}\left(<\left(\tilde{S}_{H H}-\tilde{S}_{V V}\right) \tilde{S}_{H V}^{*}>\right)}{-<\left|\tilde{S}_{H H}-\widetilde{S}_{V V}\right|^{2}>+4<\left|\tilde{S}_{H V}\right|^{2}>}\right)+\pi\right]
$$

The arctangent in (3) is computed in the range of $(-\pi, \pi)$. The orientation angle shift is mainly induced by the azimuth slope (perpendicular to the incidence plane), but it is also a function of the range slope and the radar look angle as indicated by the equation,

$$
\tan \theta=\frac{\tan \omega}{-\tan \gamma \cos \phi+\sin \phi}
$$

where $\phi$ is the radar incidence (look) angle, $\tan \omega$ is the azimuth slope, and $\tan \gamma$ is the slope in the ground range direction. For ocean surface wave measurement, range slopes is small, the following approximation can be made:

$$
\tan \omega=\tan \theta \sin \phi
$$

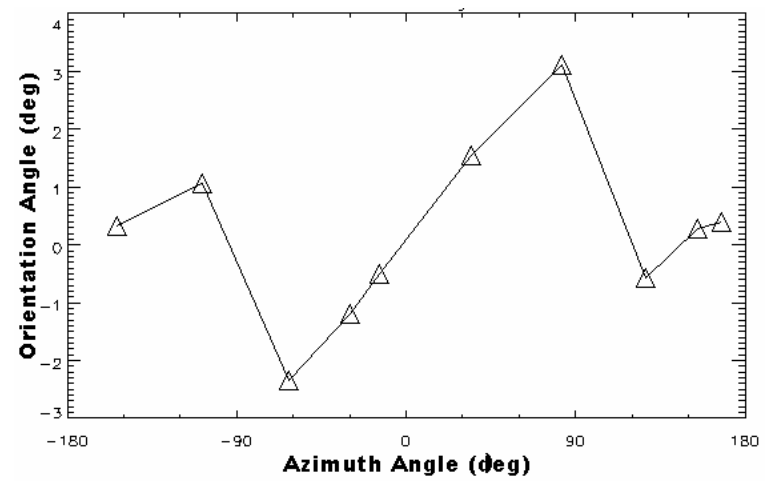

Fig. 2 Orientation angles derived from POLSCAT data.

The result is shown in Fig. 2. As expected, orientation angles show odd symmetry. The orientation angles at $0^{\circ}$ and $\pm 180^{\circ}$ are near zero due to symmetry in wind wave azimuthal slopes.

The peaks are located near $+80^{\circ}$ and $-60^{\circ}$. The peak values of $\pm 3^{\circ}$ are higher than that from L-band, but they may be correct for $\mathrm{Ku}$-band. The peaks could be used to measure wind direction without the usual $\pm 180^{\circ}$ ambiguity. Secondary peaks near $\pm 120^{\circ}$ are also observed. The same peak locations, between the orientation angles and the real part of correlation coefficient shown in Fig. 1, can be easily explained by (3) where the real parts of correlations between co-polarizations and cross-polarization are the determining factor.

The occurrence of the secondary peaks is unexpected. We theorized that the hydrodynamic modulation of small waves in the leeward slope of the long waves in the two scale model produces this phenomenon. Basically, the large POLSCAT resolution cell, 300m x 420m, contains several long waves. At the upwind direction, the reflection symmetry induces zero orientation shifts. Between $0^{\circ}$ and $\pm 90^{\circ}$, returns from leeward slopes dominate, but are partially offset by return from windward slopes, producing highest orientation shifts around $60^{\circ}$. Without significant returns from windward slopes, peaks would occur at $\pm 90^{\circ}$. At near $\pm 120^{\circ}$, the windward slopes induce higher returns, due to higher local incidence angles than those from the leeward slopes causing the reverse in 
orientation angle shifts. When the azimuth angle approaches $\pm 180^{\circ}$, reflection symmetry again makes orientation angle shifts back to zero.

We use a two-scale wave model to verify this peculiar result. The windward and leeward slopes are assumed to be equal at a value of 0.1 . To account for the hydrodynamic modulation of small waves in leeward slopes, we assume that the returns from leeward slopes are about $3 \mathrm{~dB}$ higher at the same local incidence angle. The result is given in the Fig. 3. The orientation angles based on the theoretical model matches the experiment result reasonably well.

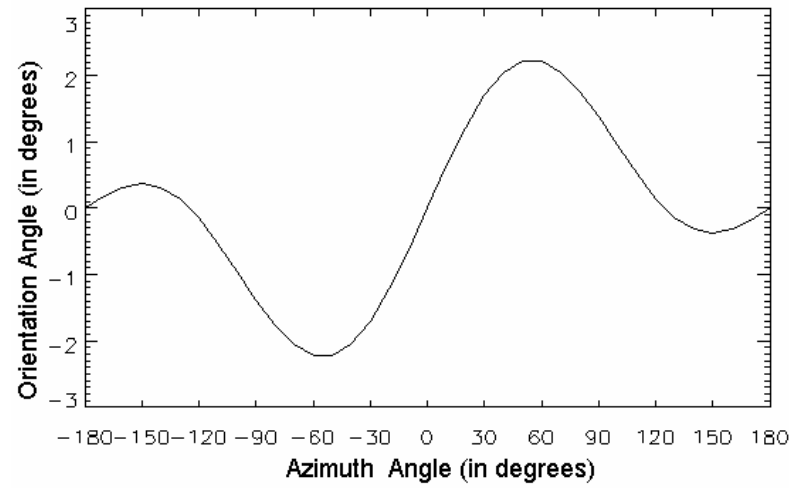

Fig. 3 Theoretical orientation angles based on a two-scale model matches the experimental results shown in Fig. 2 reasonably well.

\section{B. Depolarization effect}

To evaluate the depolarization effect as a function of radar imaging directions, we analyzed the data using the Cloude and Pottier decomposition [4]. The entropy is an indicator of randomness in scattering mechanisms. Entropy equals zero, if a single scattering mechanism is dominant, while three equally dominant scattering mechanisms produce an entropy value of 1. As shown in Fig. 4, entropy is rather high for ocean surface scatters compared with the values we have observed from Lband or C-band data (typically smaller than 0.2 ).

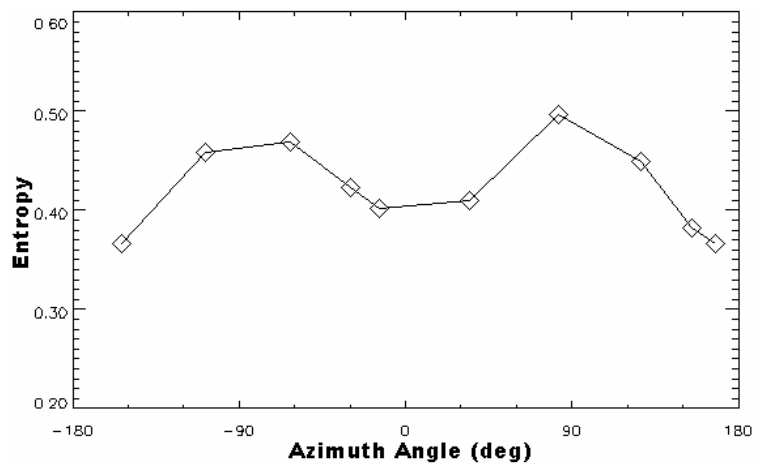

Fig. 4 Polarimetric entropy is used to evaluate the depolarization effect

The higher entropy values may be due to the inherent scattering characteristics of Ku-band. The first eigenvector has the characteristics of Bragg scattering while the other two eigenvectors have the characteristics of double bounce scattering probably due to wave breaking or foam. The heavily averaged data could also contribute toward the high

Identify applicable sponsor/s here. (sponsors) entropy values. The peaks are near $\pm 90^{\circ}$ indicating a stronger depolarization effect, because of weak echos from surface scattering at the cross wind direction that makes the contribution from double bounce terms more significant.

\section{Scattering mechanism}

Another important parameter in the Cloude and Pottier decomposition is the alpha angle, which is a measure of the type of scattering: $\alpha=0$ for specular scattering, $\alpha=45^{\circ}$ for dipole scatterings, and $\alpha=90^{\circ}$ for double bounce scatterings. For surface scattering, the typical value of alpha angle is between $10^{\circ}$ to $40^{\circ}$. The averaged alpha angle (i.e., the individual alpha values weighted by their eigenvalues) is given in Fig. 5. It shows a symmetry property as expected, and it is dominated by surface scattering. The peaks are located near $\pm 120^{\circ}$.

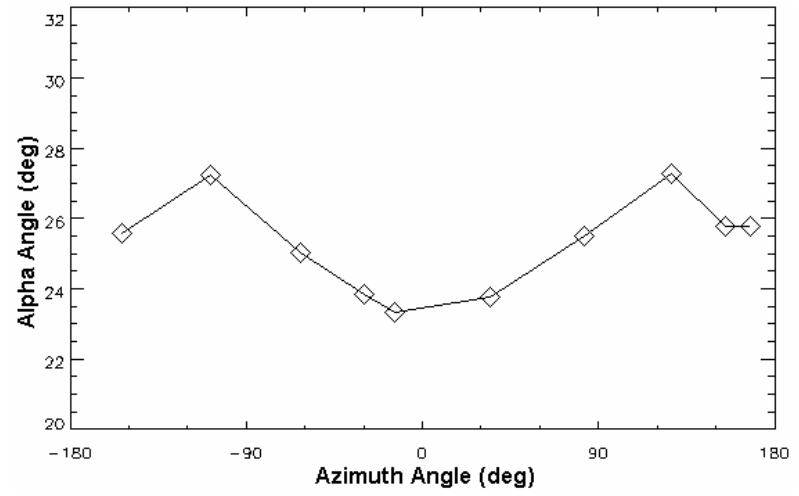

Fig. 5 Alpha angles show surface scattering dominanting for all radar look directions

\section{Range slope estimation}

Schuler et al. [5] proposed an algorithm to estimate range slopes of ocean waves based on averaged alpha angles. This algorithm is still very new, and further verification is needed. Basically, the value of the alpha angle increases with the incidence angle based on the Bragg scattering model. The alpha values for a conducting surface and the one with dielectric constant, e $=50-\mathrm{j} 40$, are shown in Fig. 6 .

If a nominal alpha value for a specified radar look angle ( $45^{\circ}$ in this experiment) can be evaluated, then the net range slope in a resolution cell, induced from the local incidence angle of long waves, can be estimated. Based on the small angle approximation, the sensitivity for a $45^{\circ}$ radar look angle is

$$
\frac{\Delta \alpha}{\Delta \theta_{i}}=\frac{\sin 2 \theta_{i}}{1+\sin ^{4} \theta_{i}}
$$

where $\boldsymbol{\theta}_{\boldsymbol{i}}$ is the local incidence angle, and $\boldsymbol{\Delta} \boldsymbol{\theta}_{\boldsymbol{i}}$ corresponds to the range slope. The nominal value of alpha angle is evaluated in this paper by the mean of all alpha values. Each alpha value is than subtracted from the mean value as an estimate of the net range slope of long waves in a resolution cell. The results are shown in Fig. 7. 


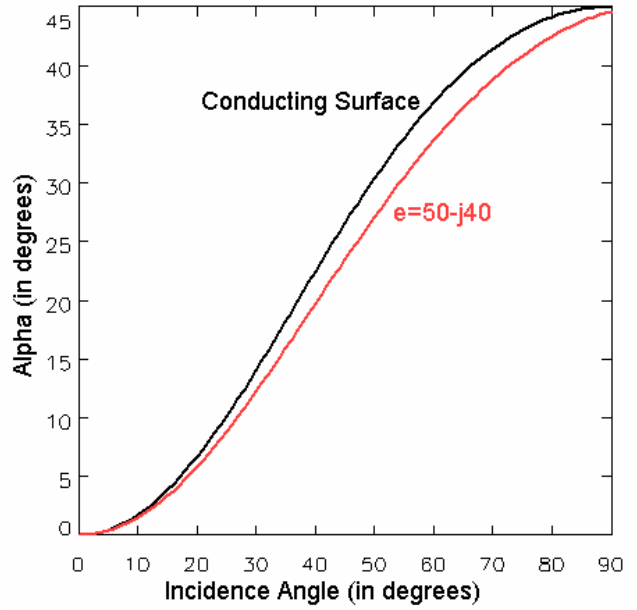

Fig. 6 Based on the Bragg scattering model, alpha angles increase with incidence angles.

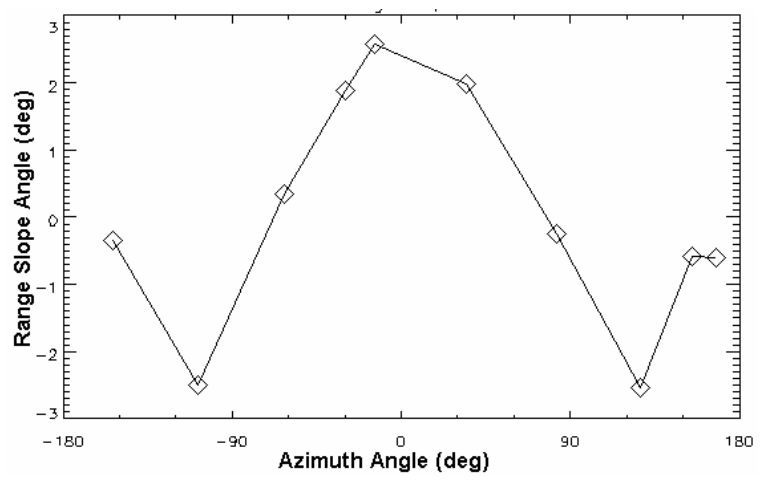

Fig. 7 The net range slope from long waves in a resolution cell are measured by alpha angles.

As expected the range slopes show even symmetry. In the upwind direction, range slopes are much higher than in the downwind direction. Secondary peaks are also shown near $\pm 120^{\circ}$. The same physical interpretation that we made for the orientation angle estimation should also be applicable here.

\section{E. Circular polarizations}

Circular polarization correlation coefficients are important parameters in polarimetric analysis, in particular, the RR and LL correlation coefficient. Fig. 8 shows correlation coefficients (coherence) of RR and LL Circular polarizations. The imaginary part (middle curve) shows odd-symmetry, but the real part (lower curve) shows even-symmetry. Its magnitude (top curve) indicates even-symmetry with a value from 0.5 to 0.7 , which is higher than expected.

\section{F. Surface roughness}

Surface roughness can be estimated by the correlation coefficient between RR and LL circular polarizations or by the anisotropy [6],

$$
\text { Anisotropy }=1-k s
$$

where $\boldsymbol{k}$ is the radar wave number, and $\boldsymbol{s}$ is RMS height. Due to the page limitation, the anisotropies is not shown, but its characteristics is similar to the magnitude of $\mathrm{RR}$ and $\mathrm{LL}$ correlation coefficients of Fig. 8 (upper curve). It has been shown that this estimation is independent of dielectric constant, and the biasing effect from long slopes. The algorithm was developed for land surface application, and verified with polarimetric SAR data of farmland. Consequently, its applicability to ocean surface is not proven and requires further studies.

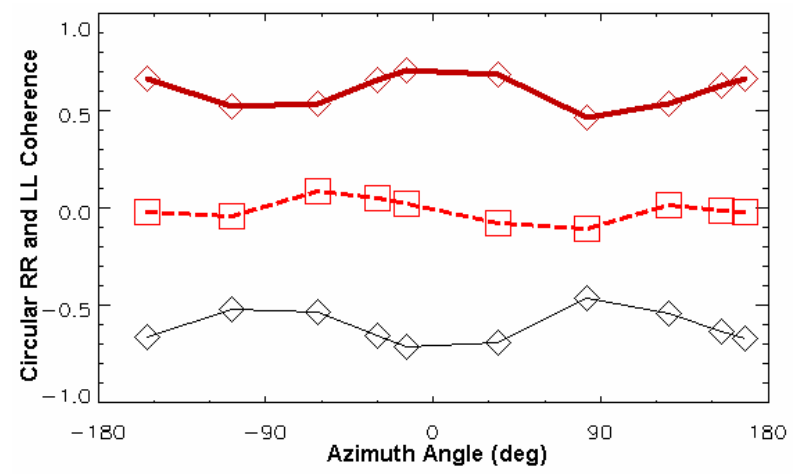

Fig. 8 Correlation coefficients (coherence) of RR and LL Circular polarizations shows odd-symmetry for the imaginary part (middle curve), but even-symmetry for the real part (lower curve). The magnitude (top curve) indicates evensymmetry with a value from 0.5 to 0.7 .

\section{CONCLUSION}

In summary, the JPL polarimetric scatterometer data has been further analyzed to study the symmetry properties based on polarimetric scattering characteristics. We believe that the newly derived scattering characteristics have the potential to solve the $180^{\circ}$ ambiguity in wind direction, and to enhance the accuracy of wind vector measurements.

\section{ACKNOWLEDGMENT}

The authors would like to thank Radar Science and Engineer Section, Jet Propulsion Laboratory, for conducting the POLSCAT experiment and made the data available to us.

\section{REFERENCES}

[1] S. H. Yueh and W.J. Wilson, "Polarimetric Radar Remote Sensing of Ocean Surface Wind," IEEE TGRS, vol.40, no. 4 April 2002.

[2] S.H. Yueh, R. Kwok, S.V. Nghiem, "Polarimetric Scattering and Emission Properties of Targets with Reflection Symmetry,' Radio Science, vol. 29 , no. 6,1994 .

[3] J.S. Lee, D.L. Schuler, and T.L. Ainsworth, "Polarimetric SAR Data Compensation for Terrain Azimuth Slope Variation," IEEE TGRS, vol. 38, no. 5, September 2000.

[4] S. R. Cloude and E. Pottier, "A Review of Target Decomposition Theorems in Radar Polarimetry," IEEE TGRS, vol. 34, March 1996.

[5] D.L. Schuler, J.S. Lee, D. Kasilingam, E. Pottier, "Measurement of Ocean Surface Slopes and Wave Spectra using Polarimetric SAR Image Data," Remote Sensing of Environment, vol. 91, no. 2, pp.197-210, May 2004.

[6] I. Hajnsek, E. Pottier, S.R. Cloude, "Inversion of Surface Parameters from Polarimetric SAR," IEEE TGRS, vol. 41, no. 4, 724-744, April 2003. 